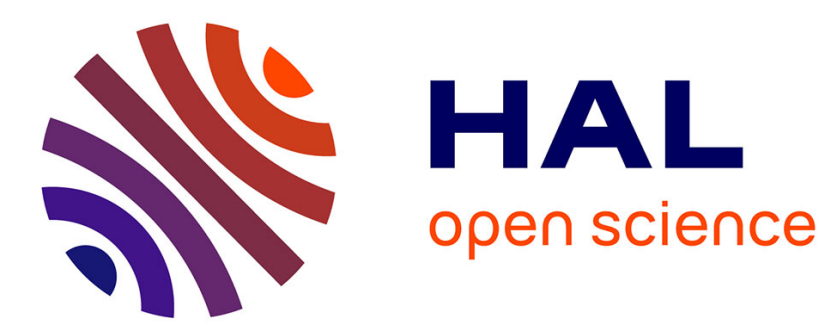

\title{
Utilisation d'une onde progressive de surface pour tableau graphique de grandes dimensions
}

\author{
S. Bigot, M. Kamarei, M. Bouthinon
}

\section{To cite this version:}

S. Bigot, M. Kamarei, M. Bouthinon. Utilisation d'une onde progressive de surface pour tableau graphique de grandes dimensions. Revue de Physique Appliquée, 1988, 23 (7), pp.1257-1263. 10.1051/rphysap:019880023070125700 . jpa-00245939

\section{HAL Id: jpa-00245939 https://hal.science/jpa-00245939}

Submitted on 1 Jan 1988

HAL is a multi-disciplinary open access archive for the deposit and dissemination of scientific research documents, whether they are published or not. The documents may come from teaching and research institutions in France or abroad, or from public or private research centers.
L'archive ouverte pluridisciplinaire HAL, est destinée au dépôt et à la diffusion de documents scientifiques de niveau recherche, publiés ou non, émanant des établissements d'enseignement et de recherche français ou étrangers, des laboratoires publics ou privés. 


\title{
Utilisation d'une onde progressive de surface pour tableau graphique de grandes dimensions
}

\author{
S. Bigot, M. Kamarei et M. Bouthinon \\ Laboratoire Electromagnétisme Micro-ondes et Optoélectronique, Unité Associée au CNRS $\mathrm{n}^{\circ} 833$, \\ ENSERG, 23 Av. des Martyrs, 38100 Grenoble, France
}

(Reçu le 19 octobre 1987, révisé le 4 janvier 1988, accepté le 15 février 1988)

\begin{abstract}
Résumé. - Le développement de la conception assistée et de la communication par ordinateur exige un nouveau type de tablettes graphiques dont les améliorations sont l'augmentation des dimensions tout en conservant une bonne précision et l'obtention d'un crayon libre de toute attache physique avec la tablette. La solution adoptée repose sur le principe de fonctionnement suivant : une onde de surface émise par le crayon pointeur se propage omnidirectionnellement dans le plan de la tablette. Trois récepteurs situés à la périphérie de la tablette captent cette onde. La mesure du déphasage en ces trois points fournit la position du pointeur.

Abstract. - The development of CAD and computer communications demands a new type of graphics tablet of larger dimensions and high precision, using a freely movable pen which is not in any way connected with the tablet. A solution is proposed where in the tablet an omnidirectional surface wave travels, which is induced by the pen. Three antennas, placed at the edges of the tablet, receive this wave. The two phase differences between the three received signals enable the calculation of the pen position.
\end{abstract}

\section{Introduction.}

Nous présentons la conception d'un dispositif plat d'affichage graphique intégrant un capteur de position qui permet de détecter et d'afficher en temps réel la trajectoire d'un pointeur sur la surface plane.

Cette description, seule, nous fait penser à une tablette graphique comme il en existe actuellement de nombreux modèles. Mais le modèle étudié, de par ses caractéristiques présentées ci-dessous, nécessite des solutions nouvelles. En plus de la définition donnée ci-dessus s'ajoutent les exigences suivantes :

* la taille du tableau doit être importante (environ $2 \times 1 \mathrm{~m}^{2}$ ) afin de permettre de nouvelles applications du type vidéoconférence ;

* la précision de localisation du pointeur doit être de 1 millimètre;

* toute liaison physique entre le pointeur et le tableau doit être éliminée.

Lorsque tous ces paramètres sont réunis (Fig. 1), les applications de ce tableau graphique deviennent nombreuses :

* toutes les applications de la table à digitaliser sont possibles (numérisation d'images) puisque notre tableau remplit au moins les mêmes fonctions : grâce

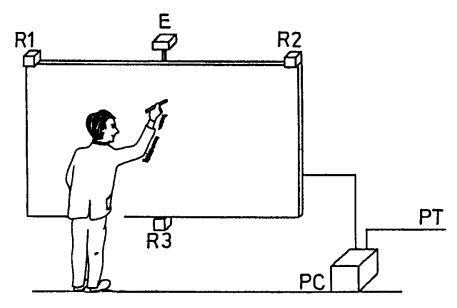

Fig. 1. - Notre tableau graphique.

[Our graphic board.]

au pointeur, on écrit réellement sur le tableau et dans le même temps on obtient sa position. Le rapport précision de localisation sur dimension $d u$ tableau est bon (1/1000) et permet donc des dessins de précision;

* le tableau est en outre utilisable pour une téléconférence. En effet, tout dessin, toute écriture tracés sur le tableau par le conférencier peuvent être retransmis n'importe où par voie téléphonique ;

* le tracé étant mémorisé dans un ordinateur, ce dernier peut commander toutes les machines qui nécessitent un patron, un modèle dessiné comme par exemple une machine de découpe automatique au laser, une machine à graver... ; 
* le tableau se transforme aisément en copy board par adjonction d'une photocopieuse.

A notre connaissance, les technologies adaptées aux tables à digitaliser ne conviennent pas du tout pour ce tableau graphique. C'est ce que nous verrons dans les paragraphes suivants en passant en revue les divers types de tables à digitaliser qui existent à l'heure actuelle sur le marché [1].

(a) La tablette mécanique. - Le pointeur est porté par deux bras articulés fixés à l'un des coins de la tablette. La mesure des angles formés par ces bras fournit la position du pointeur.

Les dimensions de cette tablette peuvent être importantes, la précision de localisation est bonne mais il existe une liaison mécanique rigide entre le pointeur et la tablette.

(b) La tablette à maillage capacitif. - C'est la plus répandue. La capacité formée par le pointeur et le maillage varie avec la position du pointeur.

La précision est bonne tant que les dimensions restent modestes $(\# 50 \mathrm{~cm})$. Dès que les dimensions deviennent importantes, une foule de problèmes apparaît. En outre, un câble souple relie le pointeur à la tablette.

(c) La tablette à rayons lumineux. - Le pointeur coupe des rayons lumineux qui forment un matriçage à la surface de la tablette, signalant ainsi sa position.

Le pointeur est entièrement libre, peut-être quelconque, mais les dimensions doivent rester faibles pour que le système ne devienne pas irréalisable.

(d) La tablette résistive. - Celle-ci est formée d'une plaque conductrice élevée à un potentiel $V$, superposée à une plaque résistive. La pression exercée par le pointeur fait apparaître un point de contact entre les deux plaques. Une mesure de la différence de potentiel aux bornes de la plaque résistive fournit la position du pointeur.

Le pointeur est libre et quelconque mais les mesures des amplitudes de tension sont entâchées d'erreur. En outre, la précision devient mauvaise pour les grandes dimensions.

Pour conclure cette revue des tablettes actuelles, on peut dire qu'aucune d'entre elles ne réunit les trois paramètres suivants :

- grandes dimensions ;

- bonne précision ;

- pas de liaison physique pointeur-table.

C'est pourquoi nous avons mis au point un système dont le principe de fonctionnement est entièrement nouveau, qui satisfait aux conditions indiquées.

\section{Principe de fonctionnement.}

On a vu que les trois paramètres à réunir se rapportent à :

Liaison - Précision - Dimension. (a) Dans le principe que nous proposons le problème de la liaison est résolu comme suit :

Le pointeur émet une onde électromagnétique qui va se propager jusqu'à trois récepteurs placés sur la périphérie du tableau. La mesure des caractéristiques de l'onde qui varient en fonction de la distance entre le pointeur émetteur et les récepteurs permet la localisation du pointeur.

Par conséquent, la liaison est réalisée par propagation d'ondes qui ne nécessite aucun autre support matériel que le tableau. Nous verrons ultérieurement l'importance du matériau formant le tableau.

(b) Pour la dimension, il suffit que le pointeur émette avec une puissance suffisante (de l'ordre de un milliwatt) pour que l'onde, après avoir parcouru la distance la séparant des récepteurs, soit encore détectée par ceux-ci. La puissance mise en jeu reste ainsi bien en deçà des normes de sécurité.

(c) Quant à la précision, il faut exclure les mesures d'amplitude qui sont peu reproductibles. Nous avons choisi la mesure de phase.

Notre système fonctionne comme suit :

L'onde émise par le pointeur parcourt la distance $d_{1}$ pour atteindre le récepteur $R_{1}$ avec une phase $\phi_{1}$ proportionnelle à $d_{1}$

$$
\phi_{1}=(2 \cdot \pi \cdot f / v) d_{1}
$$

où $v$ est la vitesse de propagation, fonction caractéristique du milieu.

De même pour $R_{2}$ et $R_{3}$ (Fig. 2). La mesure de la différence de phase $\phi_{1}-\phi_{3}$ nous permet de déduire que le pointeur se trouve sur la demi-hyperbole d'équation :

$$
d_{1}-d_{3}=(v / 2 \cdot \pi \cdot f)\left(\phi_{1}-\phi_{3}\right) .
$$

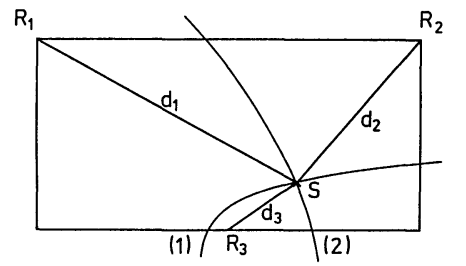

Fig. 2. - Principe de fonctionnement.

[Working principle.]

De même pour $\phi_{2}-\phi_{3}$ :

$$
d_{2}-d_{3}=(v / 2 \pi \cdot f)\left(\phi_{2}-\phi_{3}\right) .
$$

Par conséquent, le pointeur est localisé à l'intersection des deux demi-hyperboles (1) et (2) (Fig. 2). Ce système est à rapprocher de ceux utilisés en radiolocalisation.

Ce principe de fonctionnement induit les problèmes suivants :

* Plusieurs modes de propagation peuvent exister, que ce soit dans l'air ou à travers le tableau. Il est 
préférable de n'avoir qu'un mode pour n'avoir qu'une vitesse de propagation et par conséquent des mesures fiables.

Afin d'obtenir la meilleure précision possible pour la localisation de la pointe traçante, l'onde doit se propager à partir de l'extrémité du pointeur qui permet le tracé, de façon à atteindre les récepteurs en restant au niveau de la surface du tableau. On cherche à obtenir un mode de propagation en onde de surface, qui apporte en outre les deux avantages suivants :

- L'onde ne se propage que lorsque le pointeur est très proche du tableau (environ deux à trois millimètres). Il est par conséquent inutile pour l'utilisateur de manipuler un interrupteur pour signaler s'il désire réellement ou non effectuer un tracé.

- L'onde est piégée dans le tableau et de ce fait peu sensible aux parasites extérieurs (mouvements de l'utilisateur, champs externes...). Elle peut en revanche être sensible aux défauts internes du matériau mais un calibrage initial permet de contourner ce problème.

* Le principe de fonctionnement interdit la possibilité d'une horloge de référence puisque l'émetteur se trouve dans le pointeur, physiquement libre par rapport au tableau. C'est pourquoi la localisation nécessite une mesure de différence de phase entre les ondes au niveau des récepteurs, et par conséquent, un minimum de trois récepteurs.

* Une mesure de phase se fait à $360^{\circ}$ près, ce qui équivaut à dire que l'on connaît les différences des distances $d_{1}, d_{2}$ et $d_{3}$ à une longueur d'onde près. Par conséquent, si les dimensions du tableau sont supérieures à une longueur d'onde, il existera sur ce tableau plusieurs hyperboles différentes ayant la même équation. On ne saura donc pas si le pointeur se trouve sur une hyperbole ou une autre. Afin d'éliminer cette ambiguïté, on utilise une longueur d'onde suffisante, ce qui correspond à une fréquence maximale de $75 \mathrm{MHz}$. Un nouveau problème apparaît alors :

- La précision maximale de la mesure de déphasage est d'environ $2^{\circ}$, ce qui donne en distance une précision de localisation du pointeur de 2 centimètres. Cette valeur est beaucoup trop importante. Une fréquence plus élevée est indispensable pour obtenir une bonne précision. C'est pourquoi nous avons choisi que le pointeur émette une onde à $2,5 \mathrm{GHz}$, permettant la précision, modulée à $50 \mathrm{MHz}$ pour éliminer l'ambiguïté. La multiplication des deux signaux puis un filtrage passe-bas (démodulation synchrone) fournit une tension proportionnelle au cosinus du déphasage. Pour le sinus, la même opération est effectuée après avoir fait subir à l'un des signaux un déphasage de $90^{\circ}$ [2].

* L'émission depuis le pointeur pose un problème supplémentaire. En effet, pour rester maniable, léger et peu encombrant, le pointeur ne peut pas contenir un émetteur, de surcroît assez complexe puisque devant émettre une onde modulée ce qui signifie deux oscillateurs, un mélangeur, un amplificateur et l'alimentation. Nous avons résolu le problème en plaçant un émetteur principal fixé au tableau émettant une onde de fréquence $1,25 \mathrm{GHz}$ modulée à $25 \mathrm{MHz}$ se propageant dans l'air. Le pointeur reçoit cette onde par son extrémité supérieure, en double la fréquence avant de réémettre cette onde à $2,5 \mathrm{GHz}$ modulée à $50 \mathrm{MHz}$ en onde de surface. Le doubleur de fréquence intégré au pointeur est entièrement passif, simple et léger [3]. On atteint ainsi le schéma de principe de la figure 3 .

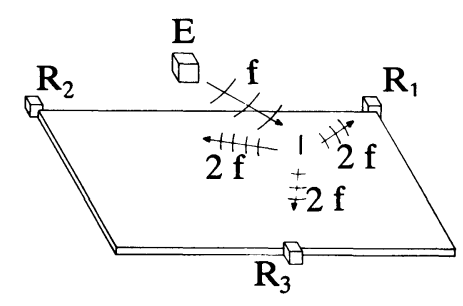

Fig. 3. - Schéma de principe.

[Principle diagram.]

* La sortie finale se fait en coordonnées rectangulaires donnant la position du pointeur. Le logiciel effectuant la conversion des mesures de déphasages en coordonnées doit impérativement être rapide pour que l'on obtienne une détection en temps réel. Cette conversion se fait par résolution d'une équation du second degré. La méthode de résolution analytique fournit une réponse immédiate.

* Le problème essentiel a été celui de rendre les ondes progressives à l'intérieur du tableau. L'onde se propage à partir du pointeur. Lorsqu'elle parvient aux bords du tableau, elle s'y réfléchit et les récepteurs ne reçoivent plus seulement l'onde directe provenant du pointeur mais également toutes les ondes réfléchies qui forment un réseau d'ondes stationnaires rendant toute mesure impossible. Il fallait donc éliminer au maximum ces réflexions sous peine de rendre ce système totalement inopérant. Nous avons essayé plusieurs solutions (Fig. 4) [4] :

(a) Nous avons construit un tableau sous forme de sandwich afin de créer des ondes progressives pour que l'onde, arrivée aux bords, n'ait plus qu'une faible amplitude (Fig. 4a). L'onde réfléchie est alors presque inexistante. Mais les réactions du sandwich sont très différentes suivant la position du pointeur.

(b) Nous avons entouré le tableau d'absorbant (Fig. 4b). Mais les absorbants utilisés étaient mal adaptés au tableau et la réflexion avait toujours lieu. Il existe d'autres types d'absorbants plus complexes 


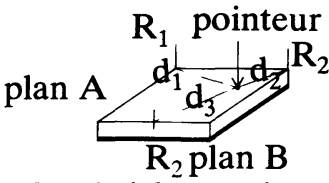

Sandwich ou plaque conductrice

a)

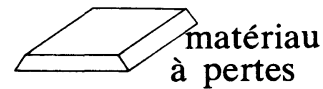

(c)

Fig. 4. - Comment éviter les réflexions ?

[How can we avoid reflections?]

et peut-être plus efficaces (profil en silicone gainé de couches de tricot de fil d'acier cuivré étamé, sphères de fer carbonyl dispersées dans une résine...), mais de prix prohibitifs.

(c) Nous avons chanfreiné les bords du tableau afin que l'onde s'évanouisse dans l'angle ainsi formé (Fig. 4c). Mais pour être efficace, le chanfrein doit avoir un angle très aigu et utilise par conséquent une grande partie du tableau, ce qui rend cette solution peu intéressante.

La solution a finalement été obtenue en entourant le tableau par un matériau composite de dimensions convenables (quart d'onde). Les réflexions sont alors fortement atténuées et les mesures possibles.

Une fois ces problèmes résolus et les fréquences choisies, il faut déterminer le matériau formant le tableau qui permet la propagation de l'onde souhaitée. Pour ce faire, une étude de la propagation des ondes de surface est nécessaire.

\section{Structure de l'onde de surface $[5,6]$.}

Par simplification, nous étudions la propagation de l'onde de surface dans la direction $z$ longitudinalement au tableau de diélectrique qu'on peut considérer comme infini puisqu'il est adapté en ses côtés (Fig. 5).

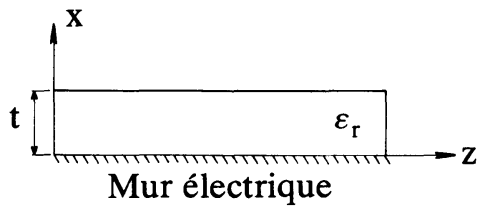

Fig. 5. - Ondes de surface suivant un diélectrique sur un plan de masse.

[Surface waves on a dielectric coated conductor.]

Les ondes de surface des deux types TE et TM peuvent être guidées le long d'une plaque diélectri- que d'épaisseur $2 . t$. Le diélectrique est supposé sans perte donc $\varepsilon_{\mathrm{r}}$ est réel. Pour les modes TM, le champ magnétique n'a qu'une composante $H_{y}$ et les composantes du champ électrique sont $E_{x}$ et $E_{z}$. Pour les modes TE, les champs magnétiques et électriques ont leurs rôles interchangés. Les composantes sont donc $E_{y}, H_{x}, H_{z}$.

La propagation des modes se fait dans la direction $z$ avec un facteur de propagation $\mathrm{e}^{-j \beta z}$. Pour les deux types de modes, le champ décroît exponentiellement dans la direction $x$ à l'extérieur du diélectrique suivant le facteur $\mathrm{e}^{-p(|x|-t)}$.

L'équation d'onde $\beta^{2}=k_{0}^{2}+p^{2}$ devant être satisfaite, la longueur d'onde et la vitesse de phase, qui sont inversement proportionnelles à $\beta$ sont toutes deux inférieures à leurs valeurs dans l'air.

Les modes TM. - Les solutions pour $H_{y}$ dans le diélectrique peuvent être du type symétrique (pair) ou antisymétrique (impair), selon la façon dont $H_{y}$ varie avec $x$ de part et d'autre du plan de symétrie $x=0$. Pour les solutions paires $\left(\delta H_{y} / \delta x\right)_{x=0}=0$ et, puisque $E_{x}$ est proportionnel à ce rapport, le champ électrique tangentiel s'annule sur le plan $x=0$.

Un plan conducteur peut être placé suivant le plan $x=0$ et la solution en résultant est un mode TM pair le long d'un diélectrique d'épaisseur $t$ placé sur un plan conducteur. Les solutions impaires correspondent à un mur magnétique suivant le plan de symétrie $x=0$.

Si la plaque diélectrique est placée sur un plan conducteur, on doit diviser par deux son épaisseur pour conserver les mêmes modes qui se propagent sur la lame diélectrique d'épaisseur 2 . $t$ placée dans l'air. On obtient alors un gain de poids et l'encombrement diminue (Fig. 5). Nous aurons alors uniquement la possibilité de modes pairs dont les composantes sont :

$H_{y}=B \cdot \cos (h \cdot x) \mathrm{e}^{-j \beta z}$ pour $|x| \leqslant t$

avec

$$
\beta^{2}=\varepsilon_{\mathrm{r}} \cdot k_{0}^{2}-h^{2}
$$

$H_{y}=A \cdot \exp [-p(|x|-t)-j \beta z]$ pour $|x| \geqslant t$

et

$$
\beta^{2}=k_{0}^{2}+p^{2} .
$$

En $x= \pm t, H_{y}$ et $E_{z}$ doivent être continus. On obtient alors :

$$
A=B \cdot \cos (h \cdot t)
$$

et $\quad \varepsilon_{\mathrm{r}} \cdot p=h \cdot \operatorname{tg}(h \cdot t)$

d'où $\quad \varepsilon_{r} \cdot p \cdot A=h \cdot B \cdot \sin (h \cdot t)$.

D'autre part, les équations en $\beta$ donnent :

$$
\left(\varepsilon_{\mathrm{r}}-1\right) k_{0}^{2}=p^{2}+h^{2} .
$$


Finalement :

$$
\begin{aligned}
\varepsilon_{\mathrm{r}} \cdot p \cdot t & =h \cdot t \cdot \operatorname{tg}(h \cdot t) \\
(p \cdot t)^{2}+(h \cdot t)^{2} & =\left(\varepsilon_{\mathrm{r}}-1\right)\left(k_{0} \cdot t\right)^{2}
\end{aligned}
$$

dont les solutions sur un plan $(h, t, p . t)$ sont les intersections entre les cercles de rayon $\left(\varepsilon_{\mathrm{r}}-1\right)^{1 / 2} \cdot k_{0} \cdot t$ et de centre $(0,0)$, et des courbes $p \cdot t=[h \cdot t \cdot \operatorname{tg}(h \cdot t)] / \varepsilon_{\mathrm{r}}$.

L'allure des courbes est donnée figure 6.

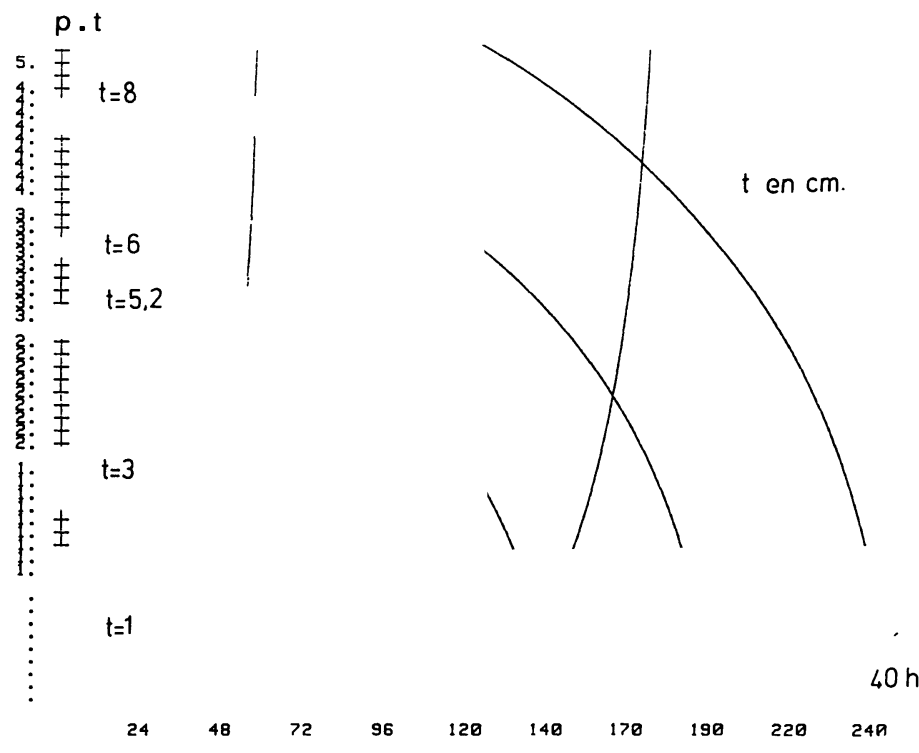

Fig. 6. - Les solutions ondes de surface.

[Solutions are surface waves.]

Les paramètres influents pour la propagation des ondes de surface sont donc:

* $\varepsilon_{\mathrm{r}}=$ la permittivité relative du diélectrique du matériau ;

* $k_{0}=2 \pi / \lambda_{0}$, proportionnel à la fréquence de travail ;

$t=$ l'épaisseur du diélectrique.

En outre, le diélectrique doit être à faibles pertes pour permettre une bonne propagation. Les diélectriques de ce type les plus courants sont le polytétrafluoréthylène (téflon), le polyéthylène et le polystyrène. Des raisons de coût et de conservation des propriétés diélectriques dans le temps nous ont conduits à choisir le polyéthylène. Sa permittivité relative est de 2,3 et pour la propagation d'une onde de surface monomode à $2,5 \mathrm{GHz}$, une épaisseur de $30 \mathrm{~mm}$ convient.

Le tracé de la figure 6 nous apporte plusieurs informations. Seules les intersections pour lesquelles $p . t$ est positif donnent des solutions ondes de surface. Par conséquent :

* Il y a intersection donc solution pour tous rapports $t / \lambda_{0}$ aussi petits soient-ils. Donc, le premier

REVUE DE PHYSIQUE APPLIQUÉE. - T. 23, N 7, JUILLET 1988 mode pair, appelé TMo, n'a pas de fréquence de coupure.

* Pour une épaisseur $t$ assez grande, on observe plusieurs modes de propagation. Tous les modes autres que TMo ont une fréquence de coupure.

Il est par conséquent possible de fonctionner en monomode. On se fixe l'utilisation du mode TMo et par là même une épaisseur du diélectrique maximale, $t_{\max }$. Cette épaisseur est telle que le rayon du cercle $\left(\varepsilon_{\mathrm{r}}-1\right)^{1 / 2} \cdot k_{0}$ soit égal à $\pi$.

Par ailleurs, pour que l'onde totale se propageant soit principalement une onde de surface, il faut que $p$ et donc $t$ soient aussi grands que possible. On doit finalement s'approcher de la valeur $t_{\max }$.

Plusieurs auteurs, Tsandoulas [10], Lo [11], Brick [12], Fernando [13], ont étudié la propagation des ondes dues à un dipôle vertical placé au-dessus d'une feuille diélectrique, elle-même collée à un plan de masse. On trouve que les champs sont composés d'une onde sphérique dans l'espace et d'une onde de surface. Cette dernière, cylindrique, possède un nombre fini de modes, nombre qu'on peut ramener à un en imposant une épaisseur du diélectrique adéquate. Il faut noter que l'amplitude des ondes sphériques s'atténue comme l'inverse de la distance à la source alors que celle des ondes de surface ne ht s'atténue que suivant l'inverse de la racine carrée de cette distance.

En appelant efficacité le rapport puissance totale émise par la source sur puissance de l'onde de surface, on obtient une valeur de plus de $80 \%$, valeur très importante.

Enfin, on calcule une hauteur d'excitation optimale qui pour nous est d'environ $3 \mathrm{~mm}$.

\section{Synoptique.}

Les différentes parties du système sont reliées comme l'indique le synoptique présenté ci-après (Fig. 7).

L'onde émise de porteuse $1,25 \mathrm{GHz}$ modulée à 25 $\mathrm{MHz}$ à partir de l'émetteur principal fixe se propage dans l'air et ce, pour trois raisons majeures :

- Aucune mesure n'est faite par l'intermédiaire de cette onde. Il n'est pas nécessaire qu'elle soit reçue au niveau de l'extrémité traçante du pointeur.

- Il n'est pas possible d'avoir une épaisseur de diélectrique et une adaptation des bords du tableau convenant à la fois pour la propagation d'une onde à $2,5 \mathrm{GHz}$ et à $1,25 \mathrm{GHz}$.

- L'onde à $1,25 \mathrm{GHz}$ ne se propageant pas dans le diélectrique ne perturbe pas l'onde qui permet les mesures, à $2,5 \mathrm{GHz}$.

Le pointeur capte cette onde à $1,25 \mathrm{GHz}$ modulée à $25 \mathrm{MHz}$ par une antenne située en son extrémité supérieure, double la fréquence et émet une onde à $2,5 \mathrm{GHz}$ modulée à $50 \mathrm{MHz}$ par son extrémité 


\section{SYNOPTIQUE}

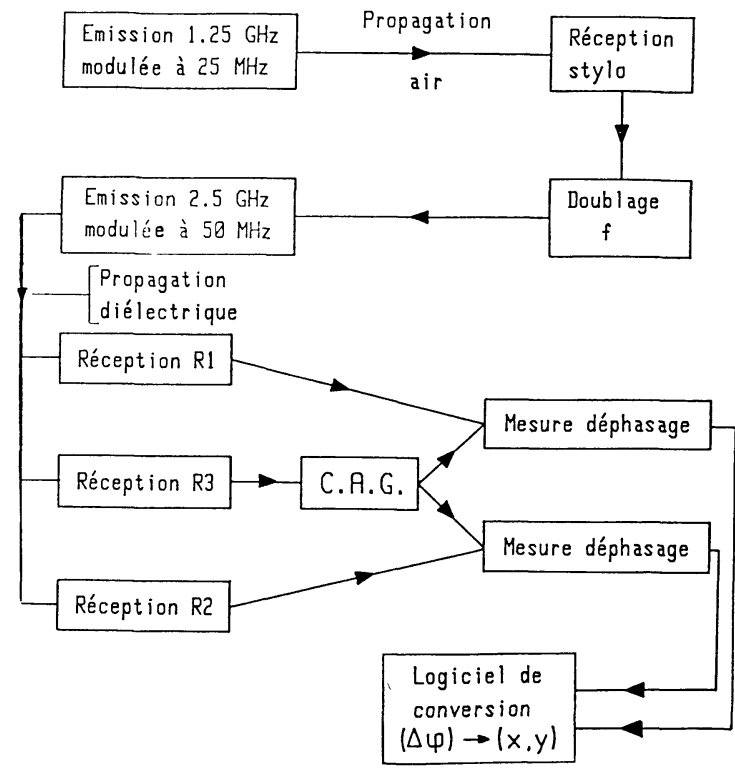

Fig. 7. - Synoptique du système complet.

[Synoptical diagram for the complete system.]

inférieure. L'onde se propage alors dans le tableau et parcourt une distance $d_{1}$ avant d'atteindre le récepteur $\mathrm{R}_{1}$. De même pour $d_{2}, \mathrm{R}_{2}$ et $d_{3}, \mathrm{R}_{3}$. Les voltmètres vectoriels mesurent le déphasage de l'onde en $R_{1}$ et $R_{3}$ et en $R_{2}$ et $R_{3}$ pour les fréquences $2,5 \mathrm{GHz}$ et $50 \mathrm{MHz}$. Ces mesures sont transmises au logiciel de conversion qui fournit les coordonnées du pointeur.

Les réalisations sont les suivantes :

Le tableau avec son adaptation de dimensions $2000 \times 1000 \times 30 \mathrm{~mm}^{3}$ et de poids $80 \mathrm{~kg}$.

Les antennes [7]:

- Une pour l'émetteur principal qui est une antenne cornet. Cette antenne est encombrante mais en contrepartie, possède une directivité optimale qui permet une illumination de tout le tableau.

- Une pour la réception à $1,25 \mathrm{GHz}$ au niveau du pointeur qui doit être à la fois légère et omnidirectionnelle dans un plan. Cette antenne est composée d'un fil de longueur $\frac{1}{4} \lambda$ placé au-dessus d'un plan de masse.

- Une pour l'émission des ondes de surface, à la base du pointeur, qui doit être fine pour que l'on ait une bonne précision de localisation, adaptée au tableau et le moins possible à l'air, omnidirectionnelle dans le plan du tableau. C'est un fil entouré d'un diélectrique taillé en pointe (antenne cierge).

- Les trois antennes au niveau des récepteurs, qui doivent être parfaitement identiques sont réalisées en technique micro ruban.

L'émetteur pilote [8] qui est constitué de deux oscillateurs Colpitt's un à $1,25 \mathrm{GHz}$ l'autre à 50 $\mathrm{MHz}$, suivis d'un coupleur, d'un modulateur à transistor puis d'un étage amplificateur.

Le doubleur intégré au pointeur qui s'inspire fortement du doubleur passif servant en particulier pour la détection des victimes d'avalanches, développé dans notre laboratoire [3]. Il a fallu le miniaturiser pour qu'il s'insère à l'intérieur du pointeur.

Les trois récepteurs [9] doivent être le plus identique possible afin de ne pas créer de déphasage supplémentaire qui fausserait la mesure. Bien sûr, pour éliminer ce risque, une calibration doit être réalisée avant la première utilisation.

De nombreux filtres à pente raide ont été réalisés pour les raisons suivantes :

- L'émetteur principal ne doit envoyer que du $1,25 \mathrm{GHz}$ (modulé à $25 \mathrm{MHz}$ ) et surtout pas d'harmonique $2(2,5 \mathrm{GHz})$ qui serait reçu par les récepteurs et nuirait à la mesure.

- Au niveau des récepteurs, après avoir amplifié le signal reçu, il faut séparer l'onde à $50 \mathrm{MHz}$ et l'onde à $2,5 \mathrm{GHz}$. On récupère la basse fréquence à l'aide d'un détecteur d'enveloppe à diode, quant à la porteuse, il faut pour la récupérer réaliser un filtre passe-bande à bande passante très étroite pour éliminer les deux raies à $50 \mathrm{MHz}$. Ensuite sont effectuées les mesures de déphasage aux deux fréquences 50 et $2500 \mathrm{MHz}$ à l'aide de 2 fois 2 voltmètres vectoriels. Ceux fonctionnant à haute fréquence ont été développés dans notre laboratoire [2].

Vient ensuite le logiciel de conversion. Comme on l'a vu auparavant, le passage de la mesure de déphasage aux coordonnées se fait par résolution d'une équation du second degré. La méthode analytique donne des résultats immédiats.

\section{Conclusion.}

Ce sujet a demandé plusieurs innovations. Il a fallu en premier lieu trouver le principe du système, puis exciter des ondes de surface à partir d'un point précis $[10,11]$ et s'arranger pour qu'il y ait peu de réflexions sur les bords. L'ensemble des circuits micro-ondes linéaires a été conçu avec l'aide du logiciel Super Compact.

Il n'est en outre pas difficile d'introduire des couleurs ainsi que l'effacement de tracés déjà effectués par jeu d'interrupteurs suivant le pointeur choisi.

La liberté du pointeur, pas de liaison physique entre celui-ci et le tableau, apporte une facilité d'utilisation appréciable. 


\section{Bibliographie}

[1] Listing des brevets Tablettes graphiques.

[2] KAMAREI, Conception et réalisation d'un dispositif de mesure de champ diffracté en vue de l'application en imagerie micro-ondes, Thèse INPG Grenoble (1985).

[3] Razban, T., Lemaitre, R., Bouthinon, M., CouMES, A., Passive transponder card system, Microwave J. 30 (octobre 1987) 135.

[4] CornbleEt, S., Multilayer structures radomes and electromagnetic windows, Microwave opt., Pure and Appl. Phys. (Academic Press inc.) 1976.

[5] Collin, Surface waves along dielectric slabs, Field theory of guided waves (McGraw Hill) 1960, p. 471.

[6] WALTER, TM dielectric sheet, Traveling wave antennas (McGraw Hill) 1965, p. 254.

[7] JASIK, Antenna engineering handbook (McGraw Hill) 1961.
[8] Farail, F. et Trione, D., Panneau de téléconférence. Rapports de D.E.A. (LEMO) septembre 1987.

[9] Bonkoungou, Conception et réalisation d'un 'système de réception faible bruit fonctionnant à 2,5 GHz, Thèse INPG Grenoble (1982).

[10] Tsandoulas, G. N., Excitation of a grounded dielectric slab by a horizontal dipole, IEEE Trans. antennas propag. Ap. $17 \mathrm{n}^{\circ} 2$ (March 1969).

[11] Lo, Y. T., Electromagnetic field of a dipole source above a grounded dielectric slab, J. Appl. Phys. 25 (June 1954) 733-740.

[12] BRICK, D. B., The radiation of a Hertzian dipole over a coated conductor, Proc. IEEE (London) 102 ser. C (1955) 104-121.

[13] Fernando, W. M.G., An investigation of the properties of radial cylindrical surface waves launched over flat reactive surfaces, Proc. IEEE (London) 103 ser. B (May 1956) 307-318. 